\title{
Research on Product Image and Home Appliance Appearance Design
}

\author{
Li Hongyan \\ Chongqing Creation Vocational College Art Department, Chongqing, 40216, China
}

Keywords: Product Image, Home Appliance, Appearance Design

\begin{abstract}
Nowadays, enterprises have fully realized the importance of industrial design to enterprise product development. This paper introduces the concept and method of product image design to help enterprises solve the instability of product style, establish the difference and contact in the same series of products, and help enterprises the establishment of the same kind of products with other enterprises in different image characteristics, thereby improving the market style of wind-style confusion.
\end{abstract}

\section{Introduction}

In today's increasingly competitive business products, it is difficult for us to distinguish products in the basic technical level or quality, the same type of products in the manufacturing process, materials, costs and other aspects of the difference is getting smaller and smaller, in most markets Different brands of goods in the function, quality and other aspects are very similar, in this case, people want to buy more is to stimulate the appearance of the product. From the NOKIA and MOTOROLA two companies in the manufacturing process, materials, it is difficult to distinguish which product quality is better, but from the appearance of the two phones I can certainly know that I need which one. This is the characteristics of the market today, technology, technology and other basic levels have been unable to meet the needs of consumers.

From the enterprise point of view, product image recognition system is more concerned about the performance of the product in the terminal, as well as with the efforts of the promotion of a good product image from the target can make enterprises have a clear market direction; from the strategic, The overall image of the enterprise; from the form, more refined, more detailed classification; from the spread, the product can be a series, the overall style to maximize the visual display in front of consumers. Thus changing the potential consumption for the actual consumption, change the occasional purchase for long-term purchase.

In addition, the establishment of the product image from the long-term interests both conducive to the establishment of corporate brand, but also conducive to product development when the product management. Enterprises need to constantly develop new products to meet the needs of customers at different times and through the establishment of the product image can be timely and accurate understanding of the customer's favorite modeling elements, and in the next development in use.

Therefore, we need to carry out in-depth study of this subject, a more profound understanding of the impact of product image theory of enterprise composition, to find the product image related elements to adapt to the product modeling aspects of the law for the company and enterprise product design guidelines direction.

\section{The Definition of Product Image}

Product image definition: "product image is a product in the minds of a variety of perceived association of mind; is the product personality and product status, characteristics, nature, etc. may not be accurate visual and other mind picture; people's views of the product, emotions and expectations. "

As a kind of image design, the concept of product image is built on the basis of the concept of image, the product as a form of carrier, must have its image (including visual image), according to the previous "image" concept We can define the concept of product image as follows: the meaning of 
product image is divided into two categories: narrow sense and broad sense: in the narrow sense: the main product itself is the image presented by the product, that is, the visual image of the product. Generalization: the general understanding and comprehensive impression of the enterprise products, it includes the product brand, function, design, technology, quality, packaging, display, advertising, marketing, use, maintenance, service and other factors, any perception of the product constitutes part of the product image.

People on the product of any ideas and external performance on the understanding of the product image constitute a part. The general meaning of the product image includes both the visual image presented by the product itself and the various associations outside the product body itself.

\section{The Main Application Areas of Product Image}

Products are the core carrier of business, but also the core of the design carrier. Product is the core tool of enterprise business competition, but also design as a material basis for the existence of a career, thus determining the product design differences in the core position. Product image design product culture is the core content, so we need to combine corporate culture, business strategy research design strategy and strategy.

\subsection{Product Visual Image.}

At the visual level, the product image is the most intuitive and general, to give users the most direct visual impact and memory, product visual image, including product form, texture and color, but also the interface shape, texture and color. Product image in the visual level of the application is the most extensive and commonly used, but also corporate and design company design focus. For example, a successful enterprise product image from the visual level, it is easy to see his product has a strong visual contact.

\subsection{Product Auditory Image.}

In the auditory level, the product image to form an indirect feeling, from the hearing to distinguish between other enterprise products, through the long-term role of hearing, leaving the user a good memory. Product auditory image includes product opening sound, key operation sound, parts open and close the sound, wonderful tone and so on. Product image in the auditory level of the application is gradually increased, such as the Nokia mobile phone comes with a special ringtones, window open music and so on.

\subsection{Product Touch Image.}

In the touch level, the product image can be relatively directly left to the consumer a deep impression on the surface of the product through the use of different materials, materials, different ways to deal with the user can bring different feelings and emotions, through prolonged contact, gradually forming a product touch image. This image is a product to bring the user's emotional image. Product image in the tactile application of the more successful is the Philips products. Through the product material changes, the application of soft material reflects the Philips people-oriented design. Through the material surface of the touch design, often can be very directly feel the grade of the product.

\section{The Applications of Product Image in the Home Appliance Product Design}

\subsection{Product Image - Consumer Communication Model.}

Product image is how to accept the consumer and how to affect the consumer's purchase behavior? This section begins with the analysis of the modeling elements of the product itself, based on the study of communication and psychology. The following figure will be the general model of communication, brand image transmission model and the brand image of the product image of the comparative analysis, the product image is the source of communication, such as Apple's product image. Information to be communicated to consumers includes: the image of the product to give 
consumers the value and functionality, Apple to convey to consumers young fashion and freedom, with Apple products reflect your fashion taste and young mentality. And the production of the product is the main carrier, the product image of the value and function is attached to the product, the other carrier may also include advertising public relations activities (commonly known as PR) and so on, and the product is the closest to consumers feel the deepest of the parts such as Apple produced ipod music player, which inherited the Apple brand concept, as Apple's carrier presented to consumers. Product image transmission mode of the recipient of course, is the consumer, and the acceptance of the consumer process analysis, it can be broken down into sensory contact, awareness, emotional understanding, until the final brand communication requirements of the target consumer to take action.

\subsection{Consumer Experience System.}

In the mass communication system, accepting information depends on its own vision, touch, hearing, smell and taste. For information dissemination of product design, the most important of which is vision, a normal person from the outside world to receive information, about $90 \%$ is obtained through the visual. Shape, color and texture through the visual stimulus function, in practice can pass more information. Most of the brand products are through these three points to convey the information.

Followed by touch and interaction between people and the product, it includes any physical contact between the user and the product, through the contact and interaction process to enhance the use of product experience, access to product confidence.

As for hearing, smell and taste, in general, less involved. However, for those products that need to be sensed by these three senses, the right sound, the right smell, and the proper taste can also enhance the consumer's good impression of the product. Such as vocal equipment, cosmetics and books need to carefully consider these three points.

The whole system and function of the system is very complex, often for the reception of information is integrated. Simply put, consumers rely on five kinds of feelings to experience the product design, the formation of information on the formation of awareness, processing and processing of some of the feelings of the product, and finally reflected in the action.

\subsection{Consumer Behavior.}

Consumer behavior is the ultimate goal of the entire communication activities, the last two parts are written by the feelings of consumers and cognitive activities, these feelings and cognitive changes, and ultimately will be put into action, reflected in their action on the product on. Marketers are used to describe these actions by approaching or leaving these two words.

Consumers have a positive impression of the product, and by the consumer will choose to close to the product after stimulation, they will buy a product to carefully observe the investigation of its specific functional parameters is not in line with their own requirements. But also compare the product with competitive products to make better choices. In this series of actions, including the product of the investigation, comparison, the purchase of products, the use of products, etc., the consumer's action status may be due to negative impression to leave.

And when the consumer has a negative feeling, he will choose to leave the product. Leaving, including the reduction of the purchase, the product on the shelves turn a blind eye to a product for negative evaluation and so on. Consumers in the use of products after the dissatisfaction with the choice to leave, is the most damaging product image, including negative evaluation, claims and so on.

Sales staff to understand the content of the product, with the product image and the nature of the product to take the correct sales behavior, so as to ensure that consumers more choices and products close.

\subsection{Consumer Awareness.}

Consumers through the system of comprehensive understanding of the product are the product image. Analysis of product image can be divided into three areas: aesthetics, usability and symbolism. The three attributes of the product together to establish consumer awareness of the product. 
Aesthetics can also be defined as whether the product is good-looking, whether it is attractive to consumers. For a long time, scholars believe that aesthetics is the most elusive, because beauty is a kind of subjective judgment from the human consciousness. There are some lines, shapes, and colors that are considered to be inherently perfect. Symmetrical right-angled geometries show the rigor of the structure, making people feel solemn, quiet, elegant, rigorous and lively rounds that make people feel inclusive, the atmosphere; relax the curve to create a dynamic shape that makes people feel free, ; Alternative avant-garde asymmetric shape with incomplete, giving a great visual impact. For each item, it has its ideal shape.

Usability can be simply defined as whether the product is easy to use. What the user needs is a comfortable and easy-to-use product. And can maintain long-term use of comfort, stability, quality and flexibility, summed up is: easy to use, safe and comfortable. Easy to use: including ease of operation and learning. Safety: the product in the use and non-use of the state or the surrounding environment will not be destroyed. Comfort: does not produce unnatural physical and psychological fatigue, and may help alleviate the fatigue. For the operational or close contact with the human body products, need to give users easy to use, safe and comfortable impression to the user to produce products or brand dependence and security.

What is symbolic and usability is that usability is the property requirement of the reaction product itself, and the symbolic nature is what the product reflects the state of the owner / user, what kind of personality is it, and what kind of social identity. Almost all of the products have a certain social symbolic meaning and the product can reflect a person or group of ideas and feelings.

The perception of the product's appearance is reflected in the process of consumption, which is the initial and perceptual understanding of the product image and brand image. This understanding is shallow and carries many uncertainties and uncertainties. Products and brands only stay in the cognitive, that products and brands at any time may lose identity. But the awareness to help establish the initial brand and its products, the initial impression, if the audience is satisfied with this point, then he will be on the brand's products will be a good impression, and if not satisfied, then he brand all products are disappointed, even if other products or other aspects of the product are very good. This is why enterprises in the promotion of products and brands to be careful, the product image, product quality, brand information and services to promote and strive to make every point to consumers' satisfaction.

\section{Conclusion}

The image of the product is a new field of enterprise image research development in recent years. The image design of the product is the refinement of the overall image goal of the enterprise. It is the system image design with the product design as the core. The basis of this study is the product image and household appliances, the combination of these two areas, the research content is mainly the methodology of the exploration, the enterprise is to rely on product survival, for enterprises, all activities are products as the core of the product to the market, accepted by consumers. The formation of product image requires a longer process, in the whole process on the one hand to maintain the image of the unity and continuity, but on the other hand must be with the development of technology, market changes or change or called innovation.

\section{References}

[1] Hou Guanhua. Product semantics in the application of product image system [J]. Friends of Science (B version). 2008 (01)

[2] Yang Ying, Lei Tian, Pan Yunhe. Product identification - a user-centric design method [J]. China Mechanical Engineering. 2006 (11)

[3] Tong Huiming. DI - New Century Industrial Design [J]. Decorative. 2002 (04)

[4] Zhang Chunhe, Fang Fang, the product image formation and clue theory research [M]. China Times Economic Publishing House, 2007

[5] Flower King Yong, the design management [M]. Beijing Institute of Technology Press, 2007 